\title{
Análisis dinámico de la estabilidad usando interpolación de alto orden
}

\section{Dynamic Stability Analysis Using High-Order Interpolation}

\author{
Pacheco-Martínez N.J. \\ Facultad de Ingeniería \\ Universidad Nacional Autónoma de México \\ Correo:nancyv9@yahoo.com.mx,nancy@unam.mx \\ Juarez-Toledo C. \\ Universidad Autónoma del Estado de México \\ Correo:toledocarlos@hotmail.com
}

Martinez-Carrillo I.

Universidad Autónoma del Estado de México

Correo:irmarcar@hotmail.com

Información del artículo: recibido: enero de 2011, reevaluado: marzo de 2011, aceptado: noviembre de 2011

\section{Resumen}

Se presenta un modelo no lineal con precisión robusta para el análisis del margen de estabilidad transitoria en sistemas eléctricos de potencia multimáquinas. Para evaluar las condiciones críticas de estabilidad dinámica, la técnica propuesta combina los métodos de interpolación de Lagrange con el de diferencias divididas de Newton, el resultado de la combinación es un método de interpolación de alto orden.

El modelo usado para aplicar la técnica corresponde al sistema interconectado de 5 -áreas de la red mexicana con 45 generadores. Como caso particular, en el contenido del trabajo se muestra la aplicación del procedimiento de alto orden para identificar el modo de baja frecuencia producido por una contingencia crítica. Ejemplos numéricos ilustran el método y demuestran la habilidad para extraer el comportamiento modal.

\footnotetext{
Abstract

A non-linear model with robust precision for transient stability analysis in multimachine power systems is proposed. The proposed formulation uses the interpolation of Lagrange and Newton's Divided Difference. The High-Order Interpolation technique developed can be used for evaluation of the critical conditions of the dynamic system.

The technique is applied to a 5-area 45-machine model of the Mexican interconnected system. As a particular case, this paper shows the application of the HighOrder procedure for identifying the slow-frequency mode for a critical contingency. Numerical examples illustrate the method and demonstrate the ability of the HighOrder technique to isolate and extract temporal modal behavior.
}

\section{Descriptores:}

- criterio de áreas iguales

- método de diferencias divididas

- método de interpolación de alto orden

\section{Keywords:}

- equal-area criterion

- Method of Divided Differences

- High Order Interpolation Method 


\section{Introducción}

El crecimiento natural de los sistemas eléctricos de potencia (SEP), las condiciones no lineales de las cargas y la interconexión entre redes ocasiona que los sistemas operen en niveles críticos de estabilidad dinámica (Fouad y Vittal, 1992). Una caracterización precisa del comportamiento dinámico del sistema de potencia en diferentes escalas de tiempo, conduce a modelos analíticos de gran dimensión donde es difícil aislar el fenómeno de interés.

Por un lado, la solución de las ecuaciones que describen el comportamiento dinámico del sistema de potencia necesitan un gran número de parámetros que varían con el tiempo y son interdependientes (Fouad y Vittal, 1992; Anderson y Fouad, 2003; Pavella, 1998). Un aspecto relevante que se ha comprobado en estudios anteriores (Juárez et al., 2006, 2005a y b; Castellanos y Juárez, 2005) corresponde al estudio del amortiguamiento de los modos de baja frecuencia (IAM, InterArea Mode), donde se ha demostrado que el buen manejo del amortiguamiento del IAM traslada las condiciones de operación inestable a un sistema más robusto.

En la literatura existen varios estudios encaminados a amortiguar las oscilaciones IAM por medio del aumento de las interconexiones entre las áreas generadas por el IAM, estas interconexiones aumentan la robustez del patrón de oscilación del modo electromecánico (Juárez, 2005a), aunque es una solución práctica, pocas veces habitual, debido al alto costo.

Para la validación de la técnica presentada se utilizaron dos sistemas de potencia, el primer modelo consta de 2 generadores con 9 líneas, conocido como modelo de prueba de 2 áreas del IEEE, el objetivo de este análisis es presentar la aplicación de la teoría expuesta. El segundo modelo corresponde a la red simplificada de la Red Interconectada Mexicana, con el propósito de validar la teoría en un modelo complejo ante condiciones de inestabilidad dinámica.

\section{Métodos de estudio empleados en el análisis de estabilidad transitoria}

El presente trabajo está encaminado al análisis del IAM, donde se demuestra que la aplicación de un modelo de reducción de sistema en los SEP puede mejorar su robustez electromecánica.

El interés por la rapidez de la determinación del análisis de estabilidad se ha reconocido extensamente en la literatura (Zhang et al., 1997). Los campos de aplicación de este tipo de formulaciones son muy variados y sobresalen la evaluación de límites de estabilidad, filtrado de contingencias, control en tiempo real y análisis del amortiguamiento instantáneo del sistema.

Usando el OMIB (One Machine Infinite Bus) dinámico (Pavella, 1998; Zhang et al., 1997) en conjunto con una herramienta no-lineal para la determinación de medidas de estabilidad (Zhang et al., 1997), se muestra una reducción significativa en el tiempo de cómputo para la obtención del margen crítico de estabilidad dinámica.

Recientemente han surgido métodos que combinan herramientas numéricas y analíticas basadas en extensiones de modelos equivalentes de dos máquinas, los cuales tienen la capacidad de aproximar la trayectoria dinámica del sistema en el periodo de posfalla y obtener ecuaciones que describan el comportamiento del sistema. A esta nueva generación de métodos de análisis pertenecen los métodos OMIB generalizados (Pavella, 1998). En este trabajo se utilizó el equivalente OMIB generalizado debido a que tiene la ventaja de reducir la cantidad de información multimáquina, conservando la información fundamental del modo electromecánico del sistema de potencia (Juárez et al., 2006).

\section{Equivalente OMIB generalizado}

Las motivaciones principales de la elección del modelo OMIB generalizado y variante en el tiempo para el desarrollo de este trabajo son características claras del método para:

- La simplificación del equivalente multimáquinas para determinar la estabilidad del sistema.

- El análisis del intercambio de energía cinética entre grupos de generadores coherentes.

- Definir los modos principales que interactúan en un disturbio y delimitar los grupos de interés.

- Seleccionar acciones que lleven al estudio, control y amortiguamiento del sistema.

El análisis de la técnica OMIB (One Machine Infinite-Bus) se utiliza ampliamente para explicar la naturaleza del problema de estabilidad transitoria. Esta técnica permite a través del denominado "criterio de igualdad de áreas" determinar si un caso es estable o inestable (Juárez et al., 2006).

A partir de un programa de estabilidad transitoria se identifican las máquinas críticas, las cuales se denotan como MC y el resto del sistema NC. Acto seguido, las variables del sistema se representan con el equivalente OMIB por medio de una ecuación de segundo orden (Juárez et al., 2005a). 


$$
M_{\text {OMIB }} \ddot{\delta}_{\text {OMIB }}=P_{m_{\text {OMIB }}}-P_{e_{\text {OMIB }}}
$$

donde el coeficiente de inercia OMIB, $M_{\text {OMIB }}$, es la combinación los momentos $(M)$ de las máquinas $i$ y $j^{1}$

$$
M_{\text {OMIB }}=\frac{\left(\sum_{i \in M C} M_{i}\right)\left(\sum_{j \in N C} M_{j}\right)}{\left(\sum_{i \in M C} M_{i}+\sum_{j \in N C} M_{j}\right)}
$$

la aceleración OMIB, $\delta_{O M I B}$, proviene de la derivación numérica del ángulo $\delta_{O M I B}$

$$
\nabla^{2}\left(\delta_{\text {OMIB }}\right)=\nabla^{2}\left(\delta_{\text {MC }}-\delta_{\text {NC }}\right)
$$

las posiciones angulares críticas y no críticas, $\delta_{M C}$ y $\delta_{N C}$ son respectivamente

$$
\delta_{M C}=\frac{1}{\sum_{i \in M C} M_{i}} \sum_{i \in M C} M_{i} \delta_{i} ; \quad \delta_{N C}=\frac{1}{\sum_{j \in N C} M_{j} ;} \sum_{j \in N C} M_{j} \delta_{j}
$$

Finalmente, $P_{e_{\text {OMII }}}$ y $P_{m_{\text {OMII }}}$ son las potencias eléctrica y mecánica, respectivamente, y quedan representadas por combinaciones de las potencias mecánicas $\left(P_{m}\right)$ y eléctricas individuales $\left(P_{e}\right)$ de las máquinas $i$ y $j$

$P_{m_{\text {OMIB }}}=M_{\text {OMIB }}\left(\frac{1}{\sum_{i \in M C} M_{i}} \sum_{i \in M C} P_{m_{i}}-\frac{1}{\sum_{j \in N C} M_{j}} \sum_{j \in N C} P_{m_{j}}\right)$,

$P_{e_{\text {OMIB }}}=M_{\text {OMIB }}\left(\frac{1}{\sum_{i \in M C} M_{i}} \sum_{i \in M C} P_{e_{i}}-\frac{1}{\sum_{j \in N C} M_{j}} \sum_{j \in N C} P_{\mathcal{e}_{j}}\right)$

Criterio de áreas iguales

El criterio de áreas iguales es una herramienta importante para determinar el tiempo crítico de los sistemas de multimáquinas (Pavella, 1998). Durante un tiempo determinado de compensación, $t_{c}$ el margen de estabili$\mathrm{dad}, \eta_{\text {OMIB }}$, asociado nos indica hasta qué punto el sistema se vuelve inestable y puede ser expresado como

$\eta_{\mathrm{OMIB}}\left(t_{c}\right)=A_{\text {des }}\left(t_{c}\right)-A_{\text {ace }}\left(t_{c}\right)$

${ }^{1}$ Note que el subíndice $i$ se utiliza para las máquinas críticas y el subíndice $j$ para las máquinas no críticas, es decir, $i \in M C$ y $j \in N C$. donde $A_{\text {ace }}$ y $A_{\text {des }}$ representan la aceleración y la desaceleración de las zonas, respectivamente. La estabilidad del sistema está garantizada si

$\eta_{\text {OMIB }}\left(t_{c}\right)>0$

El criterio de áreas iguales proporciona una medida conveniente para determinar el inicio de la inestabilidad del sistema y el margen estable asociado a la primera oscilación (Zhang et al., 1997). La derivación de los criterios simples también se generaliza a las variables en el tiempo OMIB. De la ecuación (5) tenemos:

$\eta_{\text {OMIB }}=\int_{\delta_{1}}^{\delta_{\tau}}\left(P_{m_{\text {OMIB }}}(t)-P_{e_{\text {OMIB }}}(t)\right) d \delta$

donde $\delta_{1}$ es el tiempo inicial (prefalla) del ángulo y $\delta_{z}$ es la máxima posición angular asociada a la primera oscilación después de la falla (Juárez et al., 2005a).

Un aspecto crucial es la determinación de un algoritmo eficaz para calcular el $\eta_{\text {ОмIB }}$. Refiriéndonos a la figura 1, podemos determinar el margen OMIB mediante la integración trapezoidal (Pavella, 1998) como

$$
\begin{aligned}
\eta_{\text {OMIB }}= & A_{\text {des }}\left(t_{c}\right)-A_{\text {ace }}\left(t_{c}\right) \\
= & \frac{1}{2} \sum_{k=A}^{z-1}\left(\delta_{\text {OMIB }}^{k+1}-\delta_{\text {OMIB }}^{k}\right)\left(P e_{\text {OMIB }}^{k+1}+P e_{\text {OMIB }}^{k}-2 P m_{\text {OMIB }}\right) \\
& -\frac{1}{2} \sum_{k=1}^{A-1}\left(\delta_{\text {OMIB }}^{k+1}-\delta_{\text {OMIB }}^{k}\right)\left(P e_{\text {OMIB }}^{k+1}+P e_{\text {OMIB }}^{k}-2 P m_{\text {OMIB }}\right)
\end{aligned}
$$

La figura 1 proporciona una interpretación gráfica del método del trapezoidal donde las áreas Aace y Adec tienen como límites respectivos $\left(\delta_{1}-\delta_{A}\right)$ y $\left(\delta_{A}-\delta_{z}\right)$.

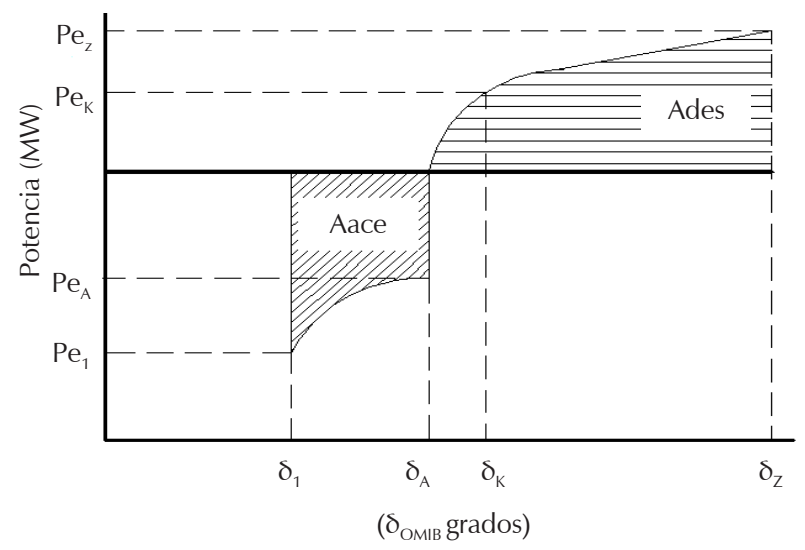

Figura 1. Representación de las áreas acelerante y desacelerante 
Al igualar a cero la ecuación (9), el margen OMIB determina la condición de estabilidad crítica respecto a un parámetro crítico de estabilidad del sistema (PCE) (Fouad y Vittal, 1992)

$\eta_{\text {OMIB }}(P C E)=0$

En las secciones siguientes se proponen varios métodos para determina el PCE.

\section{Análisis de estabilidad transitoria usando métodos lineales}

El análisis del criterio de áreas iguales (Fouad y Vittal, 1992) se fundamenta en el cálculo de la energía que el sistema de potencia adquiere en el periodo de falla (Aace) y la compara con el cálculo de la energía en el periodo de post falla (Ades).

El punto crucial del método es precisar un algoritmo eficaz e iterativo para calcular la magnitud donde ambas áreas sean iguales, nombrándolo parámetro crítico de estabilidad (PCE).

Una forma de resolver el problema es usar variantes de los métodos de bisección y de Newton Raphson (SIME). Las siguientes secciones describen la naturaleza de las aproximaciones empleadas para calcular el PCE.

Como se describe a continuación, los métodos analizados aproximan el margen de estabilidad OMIB mediante el uso de métodos numéricos para dar solución a la expresión $\eta_{\text {ОмIв }}(P C E)=0$.

\section{Método de bisección}

El método de bisección es un método lineal que se usa para resolver ecuaciones de una variable por medio del siguiente teorema:

Teorema de valores intermedios: Sea $f$ una función definida y continua en cada punto de un intervalo $[a, b]$. Si $x_{1}$ y $x_{2}$ son dos puntos cualesquiera de $[a, b]$, tales que $x_{1}<x_{2}$ y $f\left(x_{1}\right) \neq f\left(x_{2}\right)$, entonces la función $f$ toma los valores comprendidos entre $f\left(x_{1}\right)$ y $f\left(x_{2}\right)$ por lo menos una vez en el intervalo $\left[x_{1}, x_{2}\right]$.

El método de bisección parte de la noción de que si $f(x)$ es continua para $x$ entre $a$ y $b$ del intervalo $([a, b])$ y si $f(a)$ y $f(b)$ tienen signos opuestos, existe por lo menos una raíz real entre $a$ y $b$ (Juárez et al., 2005b). Aplicando el método de bisección para el cálculo del PCE se realizan los siguientes pasos:

1. Seleccionar el intervalo inicial $\left[P C E_{E}, P C E_{1}\right]$, de manera que $\eta_{\text {OMIB }}\left(P C E_{E}\right) \eta_{\text {OMIB }}\left(P C E_{I}\right)<0$
2. Calcular el primer margen de estabilidad aproximado OMIB en el punto medio entre $P C E_{E}$ y $P C I_{I}$

$P C E_{m}^{o}=\frac{P C E_{E}+P C E_{I}}{2}$

3. Usar el punto medio de la estimación, $P C E_{m^{\prime}}^{o}$ para verificar la naturaleza del margen de estabilidad:

- $\quad$ Si $\eta_{\text {OMIB }}\left(P C E_{E}\right) \eta_{\text {OMIB }}\left(P C E_{m}^{o}\right)=0$, entonces el punto aproximado de $P C E_{m}^{o}$ es el $P C E$ y el proceso está terminado.

- $\quad$ Si $\eta_{\text {OMIB }}\left(P C E_{E}\right) \eta_{\text {OMIB }}\left(P C E_{m}^{o}\right)<\mathbb{0}$, entonces $P C E_{1}=P C E_{m}^{o}$

- $\quad$ Si $\eta_{\text {OMIB }}\left(P C E_{E}\right) \eta_{\text {OMIB }}\left(P C E_{m}^{o}\right)>\mathbb{0}$, entonces $P C E_{E}=P C E_{m}^{o}$

4. Para encontrar la nueva estimación de la raíz o valor crítico se emplea

$P C E_{m}^{N}=\frac{P C E_{E}+{ }_{P C E I}}{2}$

5. Determinar la aproximación del error absoluto como

$|\epsilon|=\left|\frac{P C E_{m}^{N}-P C E_{m}^{o}}{P C E_{m}^{N}}\right| x 100 \%$

donde

$P C E_{m}^{N}=$ estimación de la iteración actual y

$P C E_{m}^{o}=$ estimación de la iteración anterior.

La convergencia se logra cuando $|\in|<=\epsilon_{s}$ es no lineal. De otra manera es necesario volver al paso 3.

El método de bisección se usa ampliamente en la industria eléctrica para determinar los PCE, debido a la sencillez de la formulación y a que garantiza la convergencia. Un efecto negativo del método de bisección corresponde a su lenta convergencia, lo que ha motivado a desarrollar métodos más rápidos que disminuyen el tiempo de procesamiento de cómputo, como el método SIME propuesto por Pavella en 1998.

\section{Método de Newton Raphson (SIME)}

El método lineal de Newton Raphson se aplica desde hace años para la evaluación del PCE en los sistemas eléctricos de potencia, también se conoce como SIME (Single Machine Equivalent) (Pavella, 1998).

La idea básica detrás del método de SIME es sustituir el comportamiento dinámico de posfalla de un sistema de energía por un equivalente OMIB dinámico en el que los parámetros se ajustan continuamente para reproducir con precisión la dinámica del sistema multi- 
máquinas. Se trata esencialmente de lograr mediante la combinación de un programa de estabilidad transitoria con el criterio extendido áreas iguales (CEAI). El cálculo de la variable en el tiempo equivalente OMIB se explica en cuatro etapas:

1. A partir de un escenario de falla dado se realiza una separación de islas del sistema, constituido por dos (o más) grupos de máquinas, a saber, el grupo crítico $(M C)$ que son las máquinas más alteradas, y el resto de las máquinas identificado como el grupo no crítico (NC).

2. Usando el centro parcial del ángulo (CPA) como marco de referencia de cada grupo, se agrega el de las máquinas de cada grupo en un equivalente.

3. Con los CPA es posible determinar la energía que se transfiere entre las islas.

4. Una transformación posterior se utiliza para representar el comportamiento dinámico del sistema de dos máquinas por un sistema equivalente OMIB en los que la trayectoria transitoria de la dinámica del sistema multimáquina está fielmente reproducida.

A continuación se exponen las ecuaciones necesarias para la aplicación del método de Newton Raphson al problema de estabilidad transitoria. Este método al igual que el método de bisección, utiliza una técnica muy eficiente para encontrar los parámetros medibles críticos de un sistema ante una contingencia.

La implementación numérica del método utiliza una secuencia de simulaciones de estabilidad transitoria para estimar el punto crítico de estabilidad. A partir de la estimación inicial dada o del valor nominal, cada solución de estabilidad transitoria se utiliza para generar un conjunto de puntos de datos $\left(P C E_{n}, \eta_{\text {OMIв }}\right)$ que se aproximan a la trayectoria OMIB para la condición de inestabilidad.

Para resolver el problema de encontrar la raíz aproximada usando el método lineal, es decir " $\eta_{\text {OMIB }}$ $\left(P C E_{i}\right)=0$ ", es necesario aproximar el conjunto de pares ordenados $\left(P C E_{k}, \eta_{\text {OMIBk }}\right)$ mediante un análisis de aproximación adecuado. Esto se implementa de la siguiente manera: dado un conjunto de $k+1$ datos de puntos, las fórmulas de interpolación de Lagrange se utilizan para encontrar un polinomio de orden $k$.

La aplicación directa se obtiene de la forma

$$
f_{n}(x)=\sum_{i=0}^{n} L_{i}(x) \eta_{\text {OMIB }}\left(P C E_{i}\right)
$$

donde $f_{n}(x)$ representa el polinomio de orden $n$ que aproxima a la función $\eta_{O M I B}$

$$
L_{i}(x)=\coprod_{\substack{j=0 \\ j \neq i}}^{n} \frac{x-x_{j}}{x_{i}-x_{j}}
$$

son funciones de ponderación que incluyen un producto de $(k-1)$ con los términos de $j=i$ omitidos. Para la aplicación numérica del método, sin embargo, es necesaria una aproximación analítica a los puntos de datos $\left(P C E_{k}, \eta_{\text {Омів }}\right)$. Detalles de la teoría del método SIME pueden consultarse en (Pavella 1998).

Basándose en un modelo de grandes sistemas y diversos escenarios de operación, dos datos de puntos $\left(P C E_{1}, P C E_{2}\right)$ (aproximación lineal) inicialmente se requieren para obtener una aproximación analítica a la condición de estabilidad crítica. El número de puntos se puede aumentar de acuerdo con el sistema.

La identificación de las MC constituye un paso crítico en el proceso. Como se señaló, el método SIME identifica la condición inestable con un programa de tiempo de dominio de estabilidad transitoria. En cada paso de la simulación los patrones de descomposición de $M C$ se consideran mediante el siguiente procedimiento de tres etapas (Juárez 2005b):

1. Clasificar las máquinas de acuerdo con sus desviaciones de ángulo del rotor.

2. Identificar las primeras grandes desviaciones angulares del rotor o las distancias entre máquinas adyacentes.

3. Seleccionar los candidatos de $M C$ de acuerdo con su mayor distancia.

El proceso continúa hasta que un grupo de candidatos de $M C$ y el correspondiente OMIB detectan condiciones de inestabilidad; después el sistema OMIB concerniente declara al candidato resultante.

\section{Método de interpolación de alto orden aplicado al concepto de estabilidad dinámica}

A continuación se propone un método sistemático para la obtención de parámetros críticos medibles de un sistema multi-máquina usando una técnica no lineal.

Para aplicar el método de interpolación de alto orden al problema de estabilidad transitoria es necesaria la síntesis de atributos instantáneos del modelo equivalente, basados en la utilización de la noción de un equivalente generalizado máquina bus infinito y el criterio de áreas iguales.

A partir de esta representación, se propone una herramienta analítica no lineal novedosa para el desarrollo y cálculo de los parámetros críticos del sistema. 
Definición 1: $n$-ésimo polinomio interpolante de Lagrange. Se define de la siguiente manera: Si $x_{0}, x_{1}, \ldots$, $x_{n}$ son $n+1$ números distintos y si $\eta_{\text {ОМ }}$ es una función cuyos valores están dados en esos números, entonces existe un único polinomio $P_{n}(x)$ de grado a lo más $n_{O M I B}$, con la propiedad de que $\eta_{\text {OMIB }}\left(P C E_{k}\right)=P\left(x_{k}\right)$ para cada $k=0,1, \ldots, n$.

Este polinomio está dado por:

$$
\begin{aligned}
& P(x)=\eta_{\text {OMIB }}\left(P C E_{0}\right) L_{n .0}(x)+. . \\
& +\eta_{\text {OMIB }}\left(P C E_{n}\right) L_{n, n}(x)=\sum_{k=0}^{n} \eta_{\text {OMIB }}\left(P C E_{k}\right) L_{n, k}(x)
\end{aligned}
$$

donde para cada $k=0,1, \ldots, n \mathrm{y}$

$$
\begin{aligned}
L_{n . k}(x) & =\frac{\left(x-x_{0}\right)\left(x-x_{1}\right) \ldots\left(x-x_{k-1}\right)\left(x-x_{k+1}\right) \ldots\left(x-x_{n}\right)}{\left(x_{k}-x_{0}\right)\left(x_{k}-x_{1}\right) \ldots\left(x_{k}-x_{k-1}\right)\left(x_{k}-x_{k+1}\right) \ldots\left(x_{k}-x_{n}\right)} \\
& =\coprod_{\substack{i=0 \\
i \neq k}}^{n} \frac{\left(x-x_{i}\right)}{\left(x_{k}-x_{i}\right)}
\end{aligned}
$$

Por simplificación en el artículo escribiremos $L_{n} \bullet_{k}(x)$ simplemente como $L_{k}(x)$ debido a que en el contenido del trabajo se expandió hasta el segundo orden. Una forma más eficiente registrada en la literatura (Wu et al., 2003) para generar el polinomio interpolante es utilizar diferencias divididas como a continuación se describe.

Supongamos que $P_{n}(x)$ es el $n$-ésimo polinomio de Lagrange que concuerda con la función $n_{\text {OMIB }}$ con los siguientes parámetros críticos de estabilidad (PCE) distintos $\left(P C E_{0}, P C E_{1}, \ldots, P C E_{n}\right)$. Las diferencias divididas de $n_{\text {OMIB }}$ respecto a los parámetros $\left(P C E_{0}, P C E_{1}, \ldots, P C E_{n}\right)$ se usan para expresar el polinomio $P n(x)$ en la forma

$$
\begin{aligned}
& P_{n}(x)=a_{0}+a_{1}\left(x-P C E_{0}\right)+a_{2}\left(x-P C E_{0}\right)\left(x-P C E_{1}\right) \\
& +\ldots+a_{n}\left(x-P C E_{0}\right)\left(x-P C E_{1}\right) \ldots\left(x-P C E_{n-1}\right)
\end{aligned}
$$

Un manejo adecuado de la ecuación (18) transforma el problema de determinar los coeficientes $a_{0}, a_{1}, \ldots, a_{n}$ por medio de un sistema algébrico de $n$ ecuaciones con $n$ incógnitas donde es fácil determinar la primera de las constantes.

Para determinar $a_{0}$ note que si $P_{n}(x)$ está escrito en la forma de la ecuación (18), entonces al evaluar $P_{n}(x)$ en $\left(P C E_{0}\right)$ sólo queda el término constante $a_{0}$; es decir, $a_{0}=P_{n}\left(P C E_{o}\right)=\eta_{\text {ОмIB }}\left(P C E_{o}\right)$. De manera similar, cuando se evalúa el $P_{n}(x)$ en $\left(P C E_{1}\right)$, los únicos términos distintos de cero en la evaluación de $P_{n}\left(P C E_{1}\right)$ son los términos constante y lineal, este procedimiento se formaliza en la siguiente ecuación.

$$
\begin{aligned}
& f\left(P C E_{0}\right)+a_{1}\left(x-P C E_{0}\right)=P_{n}\left(P C E_{1}\right)=f\left(P C E_{1}\right) ; \\
& \frac{f\left(P C E_{1}\right)-f\left(P C E_{0}\right)}{P C E_{1}-P C E_{0}}
\end{aligned}
$$

Siguiendo el trabajo descrito en (Wu et al., 2003) una forma práctica de describir el polinomio interpolante de la ecuación (18) es

$$
\begin{aligned}
& P_{n}(x)=f\left[P C E_{0}\right]+f\left[P C E_{0}, P C E_{1}\right]\left(x-P C E_{0}\right) \\
& +a_{2}\left(x-P C E_{0}\right)\left(x-P C E_{1}\right)+\ldots \\
& +a_{n}\left(x-P C E_{0}\right)\left(x-P C E_{1}\right) \ldots\left(x-P C E_{n-1}\right)
\end{aligned}
$$

donde $f\left[P C E_{0}\right]$ es la diferencia dividida cero de la función $f$ respecto a $\left(P C E_{0}\right)$ y $f\left[P C E_{0}, P C E_{1}\right]$ es la diferencia dividida uno de la función $f$ respecto a $\left(P C E_{0}, P C E_{1}\right)$, para la diferencia dividida cero de la función $f$ respecto a $P C E_{0}$, es simplemente el valor de $f$ en $P C E_{0}$

$f\left[P C E_{0}=f\left(P C E_{0}\right)\right]$

mientras que las diferencias divididas de primer orden se expresan en forma inductiva utilizando la diferencia inductiva anterior

$f\left[P C E_{0}, P C E_{1}\right]=\frac{f\left[P C E_{1}\right]-f\left[P C E_{0}\right]}{P C E_{1}-P C E_{1}}$

Un caso particular de la ecuación (18) para los siguientes pares ordenadas $\left(P C E_{1}, \eta\left(P C E_{1}\right)\right)$ y $\left(P C E_{2}, \eta\left(P C E_{2}\right)\right)$ será

$P_{1}(x)=a_{0}+a_{1}\left(x-P C E_{1}\right)$

donde es fácil demostrar que el coeficiente

$a_{2}=\frac{\eta\left(P C E_{2}\right)-\eta\left(P C E_{1}\right)}{P C E_{2}-P C E_{1}}$

Un método iterativo general, resulta al suponer que el polinomio $P_{n-1}(x)$ interpola una cantidad $n$ de pares ordenados $\left(P C E_{n}, \eta\left(P C E_{n}\right)\right)$

$$
\begin{aligned}
& P_{n-1}(x)=a_{0}+a_{1}\left(x-P C E_{1}\right)+a_{2}\left(x-P C E_{1}\right)\left(x-P C E_{2}\right)+\ldots \ldots . . \\
& +a_{n}\left(x-P C E_{1}\right) \ldots .\left(x-P C E_{n-1}\right)
\end{aligned}
$$

donde

$a_{3}=\frac{\frac{\eta\left(P C E_{3}\right)-\eta\left(P C E_{2}\right)}{P C E_{3}-P C E_{2}}-\frac{\eta\left(P C E_{2}\right)-\eta\left(P C E_{1}\right)}{P C E_{2}-P C E_{1}}}{P C E_{3}-P C E_{1}}$ 
Observe que la ecuación (26) puede simplificarse considerando (24) de la siguiente forma

$a_{3}=\frac{\frac{\eta\left(P C E_{3}\right)-\eta\left(P C E_{2}\right)}{P C E_{3}-P C E_{2}}-a_{2}}{P C E_{3}-P C E_{1}}$

de la ecuación (26) se deduce una forma práctica para definir $\mathrm{a}_{\mathrm{n}}$ en la ecuación (27)

$a_{3}=\frac{\frac{\eta\left(P C E_{n}\right)-\eta\left(P C E_{n-1}\right)}{P C E_{n}-P C E_{n-1}}-a_{n-1}}{P C E_{n}-P C E_{1}}$

\section{Algoritmo computacional}

La identificación de los polinomios de Lagrange aplicados a la determinación del PCE se realiza mediante el método de diferencias divididas con modificaciones orientadas a su determinación numérica.

El siguiente procedimiento adoptado se utiliza para determinar los polinomios de Lagrange ${ }^{2}$

1. Se proponen dos puntos iniciales $\left(P C E_{1}, P C E_{2}\right)$

2. Usando (1) se configuran los modelos OMIB generalizados en los puntos iniciales $\left(P C E_{1}, P C E_{2}\right)$.

3. Se obtienen sus respectivos pares ordenados $\left(P C E_{1}\right.$, $\left.\eta\left(P C E_{1}\right)\right)$ y $\left(\left(P C E_{2}, \eta\left(P E E_{2}\right)\right)\right.$ usando la ecuación (9).

4. Una vez determinados los pares ordenados $\left(\mathrm{PCE}_{1}\right.$, $\left.\eta\left(P C E_{1}\right)\right)$ y $\left(P C E_{2}, \eta\left(P C E_{2}\right)\right)$ se procede a calcular el polinomio Lagrange de grado uno $P_{1}(x)$.

5. Se resuelve $P_{1}\left(P C E_{3}\right)=0$ y el resultado será nuestra primera aproximación de $P C E_{3}$.

6. Se evalúa $\eta\left(P C E_{3}\right) \leq \varepsilon_{p}$ si es menor que un error predefinido $\varepsilon_{\mathrm{p}}$ entonces el algoritmo termina, en caso contrario, se vuelve a calcular el polinomio interpolante $P_{n-1}(x)$ de Lagrange.

El ciclo se repite hasta determinar algún parámetro crítico de estabilidad, $P C E_{n}$, donde $\eta\left(P C E_{n}\right) \leq \varepsilon_{p}$.

El modelado y análisis de oscilaciones electromecánicas lentas ha constituido un área de especial interés en la aplicación de métodos de análisis basados en equivalentes de dos generadores, debido a que según Juárez (2005b) el manejo eficiente de esta información puede llevar a mejorar el amortiguamiento del sistema.

\footnotetext{
${ }^{2}$ Como se expresa en la sección "criterio de áreas iguales" una manera práctica de obtener el PCE es resolver la siguiente ecuación $\eta_{\text {OMIB }}\left(P C E_{i}=0\right)$. Para casos prácticos la función $\eta_{\text {OMIB }}$ se aproxima mediante alguna función como se expone en el algoritmo.
}

A continuación se explora la aplicación de los métodos de estudio de dos generadores al análisis y la determinación de los PCE.

\section{Resultados numéricos}

Para aplicar la teoría propuesta se seleccionaron dos modelos de prueba. El sistema de prueba de dos áreas, cuatro generadores con modelo de cuarto orden del IEEE.

Las fallas a través del la línea 5-6 tienen la característica de excitar el modo interárea de menor frecuencia, separando al sistema en 2 áreas bien definidas como se muestra en la figura 2.

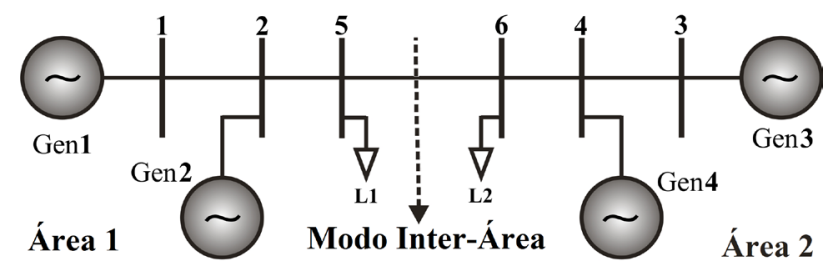

Figura 2. Sistema de dos áreas, cuatro generadores del IEEE

Para validar la teoría propuesta en un sistema complejo se utilizó el sistema interconectado mexicano (SIM) integrado por 6 áreas, 45 generadores y 238 buses, el sistema SIM fue elegido para su estudio debido a su configuración, ya que exhibe una cantidad importante de modos electromecánicos. El disturbio seleccionado fue un incremento de carga presente entre Puebla (PBD) y Minatitlán (MID), las fallas en esta línea hacen que el modo electromecánico Centro-Sureste exhiba oscilaciones electromecánicas pobremente amortiguadas como se observa en la figura 3.

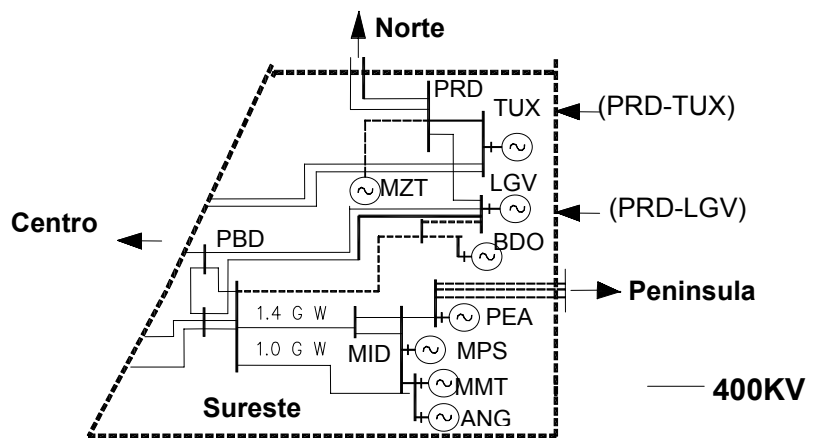

Figura 3. Sección Sureste del sistema SIM 
Solución numérica en el dominio del tiempo (sistema de dos áreas del IEEE)

Las oscilaciones electromecánicas interárea tienen su origen físico en el intercambio de energía cinética entre grupos de generadores (Zhongt y Chung,1995). En este contexto, el modelo OMIB representa el comportamiento del sistema de potencia mediante un modelo equivalente de dos generadores; el intercambio de energía entre estos dos generadores proporciona información específica sobre el intercambio de energía cinética del sistema y la naturaleza del proceso oscilatorio. Con objeto de ilustrar estas ideas, suponga que el comportamiento instantáneo del sistema se expresa en la siguiente forma

$$
\mathrm{E}_{\mathrm{C}_{\text {omib }}}(\mathrm{t})=\frac{1}{2} \mathrm{M}_{\text {omib }}(\mathrm{t}) \omega_{\text {omib }}^{2}(\mathrm{t})
$$

La ecuación (29) describe, en este análisis, las relaciones energéticas de interés para el estudio de interacción entre los dos grupos equivalentes. Una vez que se ha determinado el modelo OMIB, estas relaciones se utilizan para cuantificar la magnitud y características del fenómeno interárea, siendo uno de los tópicos más complejos y de mayor utilidad la determinación del parámetro crítico de estabilidad (PCE).

Para el análisis descrito en Pavella (1998), Juárez 2005a; Maya y Espinosa (2004) y Pacheco et al. (2010), se conjetura que para mejorar los modos de oscilación excitados por la falla, es necesario conocer los PCE con una tolerancia de error baja y lo más rápido posible para coordinar las acciones de control. Con este objetivo se discute a continuación la aplicación de diversas técnicas de análisis para la determinación de PCE usando técnicas no lineales comparándolas con métodos convencionales de tipo lineal.

Para el caso uno, se aplicó una falla trifásica variando el tiempo crítico de liberación de falla como parámetro critico de estabilidad (PCE). Estudios anteriores (Martínez et al., 2007a y 2007b) han demostrado que las fallas en esta línea excitan el modo electromecánico 7-8 con valores propios conjugados de $-1.335 \pm j 8.157^{3}$. Una de las características del comportamiento del factor de participación de los modos conjugados 7-8 es una clara división del sistema en 2 áreas, como se muestra en la figura 2.

Utilizando el procedimiento descrito en las secciones III y IV se aplicó una falla trifásica excitando los modos 7-8 del sistema de 2 áreas del IEEE los resultados de los Métodos de Bisección, SIME y Lagrange de alto orden se resumen en la tabla 1.

Se puede observar claramente en la tabla 1 que cuando el sistema se encuentra excitado en los modos 7-8 la cantidad de interacciones necesarias para determinar el PCE se reduce cuando se aplica el método de Lagrange de alto orden, logrando un menor tiempo de cómputo. La rapidez de aproximación del PCE es fundamental para determinar la acción correctiva y coordinar acciones preventivas que lleven a un estado de robustez del sistema de potencia ante fallas en las regiones críticas del sistema.

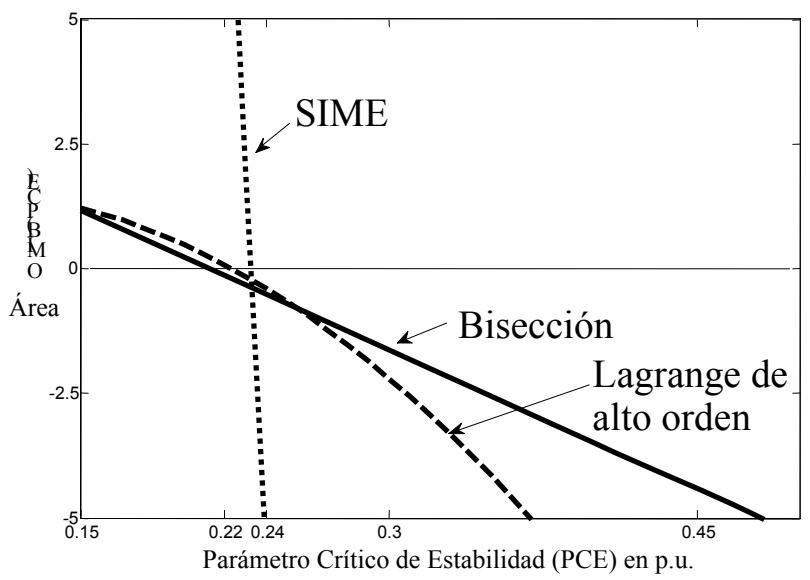

Figura 4. Convergencia de los tres métodos mostrando la no linealidad de Lagrange de alto orden

Con objeto de verificar la naturaleza de convergencia de estos métodos y la precisión con que el modelo OMIB identifica el comportamiento dinámico de interés, se graficaron los tres métodos en la figura 4 , utilizando la función final de aproximación de la tabla 1.

\footnotetext{
${ }^{3}$ Martinez I. et al., (2007a y 2007b) proponen una técnica no lineal para clasificar los modos interárea y locales del sistema de 2 áreas del IEEE.
}

\begin{tabular}{lcccccc}
\hline \multicolumn{1}{c}{ Método } & Tipo & Iteraciones & $\eta_{\text {OMIB }}(P C E)$ & $P C E$ & $\begin{array}{c}\text { Función } \eta_{\text {OMIB }}(P C E) \\
\text { Final de aproximación }\end{array}$ & $\begin{array}{c}\text { Tiempo de } \\
\text { computo }\end{array}$ \\
\hline $\begin{array}{l}\text { Bisección } \\
\text { SIME }\end{array}$ & Lineal & 12 & -0.3985 & 0.2331 & $180.72-777(\mathrm{PCE})$ & $398.31 \mathrm{seg}$ \\
$\begin{array}{l}\text { Lagrange de alto } \\
\text { orden }\end{array}$ & Lineal & 5 & -0.3838 & 0.2327 & $3.9405-18.5783(\mathrm{PCE})$ & $112.53 \mathrm{seg}$ \\
& No lineal & 4 & -0.2212 & 0.2327 & $1.0256+13.337(\mathrm{PCE})-80.34(\mathrm{PCE})^{2}$ & $88.72 \mathrm{seg}$ \\
\hline
\end{tabular}

Tabla 1. Resumen de los tres métodos aplicados a la obtención del PCE en el sistema de dos áreas del IEEE 


\begin{tabular}{lcccccc}
\hline \multicolumn{1}{c}{ Método } & Tipo & Iteraciones & $\eta_{\text {OMIB }}(P C E)$ & $P C E$ & $\begin{array}{c}\text { Función } \eta_{\text {OMIB }}(P C E) \\
\text { Final de aproximación }\end{array}$ & $\begin{array}{c}\text { Tiempo de } \\
\text { computo }\end{array}$ \\
\hline Bisección & Lineal & 7 & -0.8649 & 0.30 & $-47.5971+161.54($ PCE) & $253.14 \mathrm{seg}$ \\
$\begin{array}{l}\text { SIME } \\
\begin{array}{l}\text { Lagrange alto } \\
\text { orden }\end{array}\end{array}$ & Lineal & 5 & -0.5380 & 0.3107 & $-49.38+157.20($ PCE) & $150.04 \mathrm{seg}$ \\
\hline
\end{tabular}

Tabla 2. Resumen de los tres métodos aplicados a la obtención del PCE en el sistema al SIM

\section{Resultados numéricos del SIM}

Para el segundo caso se utilizó un sistema complejo con el SIM de 5 Áreas y tres modos electromecánicos de interés, cuyas frecuencias son $\mathrm{M}_{1}=0.34 \mathrm{~Hz}, \mathrm{M}_{2}=0.52 \mathrm{~Hz}$ y $\mathrm{M}_{3}=1.05 \mathrm{~Hz}$

El sistema analizado tiene 45 máquinas, 248 buses, 234 líneas de transmisión y 8 dispositivos SVCs.

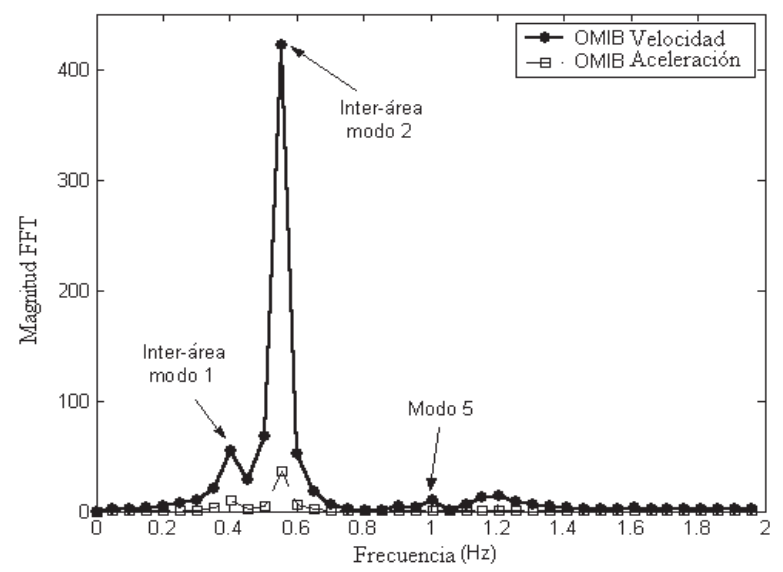

Figura 5. Convergencia de los tres métodos mostrando la no linealidad de Lagrange de alto orden

Una visualización adecuada de los modos que interfieren en la falla seleccionada se logra por medio del análisis del espectro de frecuencia en la señal de velocidad o aceleración del OMIB (figura 5).

En la figura 5 se observa que el aumento de carga en la línea PBD-MID provoca una excitación importante del modo inter-área 2 con frecuencia aproximada de 1.0 Hz. El patrón de oscilación de este modo corresponde al sistema Grijalva (PEA, MPS, MMT y ANG) oscilando en contra fase del sistema central (detalles de los patrones de oscilación de los modos electromecánicos del SIM se encuentran en Juárez (2005a)).

Finalmente, en la tabla 2 se observa claramente la reducción del tiempo de cómputo para determinar el PCE ante un sistema complejo, indicando los beneficios computacionales que lleva la aplicación de la técnica no-lineal con respecto a los métodos lineales convencionales.

\section{Conclusiones}

Se presentó una combinación del método de interpolación de Lagrange y el de diferencias divididas para obtener un método de alto orden, con la capacidad de evaluar márgenes y medidas de estabilidad (PCE). Para aplicar el modelo no lineal es necesario emplear el concepto de reducción de máquinas coherentes, concretamente el modelo OMIB.

Los estudios de simulación se llevaron a cabo para comparar y analizar el rendimiento de la propuesta del algoritmo de interpolación de Lagrange contra los métodos convencionales lineales (SIME y bisección). La aplicación eficaz de este método se puntualizó en las tablas 1 y 2.

Un resultado importante es la disminución del tiempo de cómputo para determinar los parámetros críticos de estabilidad, con lo que es posible coordinar de mejor forma los dispositivos Facts, este punto es crucial y corresponde a una extensión para un trabajo futuro.

\section{Referencias}

Anderson P.M., Fouad A.A. Power System Control and Stabilility, 2nd ed., Nueva York, John Wiley, 2003.

Castellanos B.R, Juarez T.C, R.A. Quantifying the Stability Robustness of Power Systems Using Structured Singular Value Theory, en: IEEE Power Engineering Society General Meeting, San Francisco, California EUA, 2005.

Fouad A.A., Vittal V. Power System Transient Stability Analysis Using the transient Energy Function Method, Nueva Jersey, Prentice Hall, 1992, pp. 158-162.

Juarez T.C, Castellanos B.R, Messina R.A. Analysis of Inter-Area Oscillations Using Time-Varying One-Machine Infinite Bus Equivalents, en: IEEE Power Engineering Society General Meeting, San Francisco, California EUA, 2005a.

Juarez T.C., Castellanos B.R., Messina R.A. A Higher-Order Newton Method Approach to Computing Transient Stability Margins, en: IEEE Power Engineering Society General Meeting, San Francisco, California EUA, 2005b.

Juarez T.C., Messina R.A., Vega R.D. Analysis and Control of the Inter-Area Mode Phenomenon Using Selective One-Machine Infinite Bus Dynamic Equivalents. Electric Power Systems Research, volumen 76 ( número 1), enero de 2006:180-193. 
Martínez I., Messina A.R., Vittal V. Normal Form Analysis of Complex System Models: A Structure-Preserving Approach. IEEE Transactions on Power Systems, volumen 22 (número 4), noviembre de 2007: 1908-1915.

Martínez C.I., Barocio E., Messina A.R. A Structure-Preserving Approach to Power System Normal Form Analysis, en: Power Tech 2007, Lausanne, Suiza, julio de 2007.

Maya P., Espinosa-Perez G. Output Feedback Excitation Control of Synchronous Generators. International Journal of Robust and Nonlinear Control, volumen 14 (números 9-10), junio-julio de 2004: 879-890.

Pacheco M.N.J., Juárez T.C., Martínez-Carrillo I. Análisis y control de la energía cinética transferida en el modo inter-área usando una representación máquina Bus infinito con PSSs variantes en el tiempo, en: 4o Congreso Iberoamericano de Estudiantes de Ingeniería Eléctrica (IV CIBELEC 2010), Mérida-Venezuela, 2010.

Pavella M. Generalized One-Machine Equivalents in Transient Stability Studies. IEEE Power Engineering Review (número 18), enero de 1998: 50-52.

Wu J.K., Long J., He F., He Q.L Numerical Differentiation Based Algorithm for Power Measurement, en: Fifth International Conference on Power Electronics and Drive Systems, noviembre de 2003, volumen 1, pp. 302-307.
Zhang Y., Wehenkel L., Rousseaux P., Pavella M. SIME: a Hybrid Approach to Fast Transient Stability Assessment and Contingency Selection. Electrical Power and Energy Systems, volumen 3 (número 19), 1997:195-208.

Zhongt Da.F., Chung S.T, A.K.D. Inter-Area Mode Transient Stability Estimation. Electrical Power and Energy Systems, volumen 5 (número 17), octubre de 1995: 305-312.

\section{Este artículo se cita: \\ Citación Chicago}

Pacheco-Martínez, Nancy Jacqueline, Carlos Juárez-Toledo, Irma Martínez-Carrillo. Análisis dinámico de la estabilidad usando interpolación de alto orden. Ingeniería Investigación y Tecnología XIII, 04 (2012): 451-460.

\section{Citación ISO 690}

Pacheco-Martínez N.J., Juárez-Toledo C., Martínez-Carrillo I. Análisis dinámico de la estabilidad usando interpolación de alto orden. Ingeniería Investigación y Tecnología, volumen XIII (número 4), octubre-diciembre 2012: 451-460.

\section{Semblanza de los autores}

Nancy Jacqueline Pacheco-Martínez. Graduada como maestra en sistemas eléctricos de potencia por la Universidad Nacional Autónoma de México (UNAM) en 2010, actualmente está adscrita como profesora en el programa de ingeniería eléctrica de la Facultad de Ingeniería de la UNAM, México.

Carlos Juárez-Toledo. Graduado de maestría y doctorado por el Cinvestav, México en 2003 y 2008, respectivamente. Tiene una estancia doctoral en Northeastern University, Boston, en 2007 y una estancia Posdoctoral en la UNAM en 2008. Actualmente está adscrito como profesor de tiempo completo de la UAEMex, México.

Irma Martínez-Carrillo. Graduada de maestría y doctorado en sistemas eléctricos de potencia por el Cinvestav, México, en 2002 y 2003, respectivamente. Fue ganadora del primer lugar en el Concurso Nacional de Tesis de Maestría y Doctorado. Actualmente trabaja como profesora de tiempo completo para la UAEMex, México. 